\title{
Matrix-seeded growth of nitride semiconductor nanostructures using ion beams
}

\author{
X. Weng, W. Ye, S. J. Clarke, and R. S. Goldman ${ }^{a}$ \\ Department of Materials Science and Engineering, University of Michigan, Ann Arbor, Michigan 48109 \\ V. Rotberg \\ Department of Nuclear Engineering and Radiological Sciences, University of Michigan, Ann Arbor, \\ Michigan 48109
}

\author{
A. Daniel and R. Clarke \\ Applied Physics Program, University of Michigan, Ann Arbor, Michigan 48109
}

(Received 30 June 2004; accepted 15 November 2004; published online 1 March 2005)

\begin{abstract}
We have examined the matrix-seeded growth of narrow-gap nitride nanostructures in nitrogen ion implanted GaAs and InAs. Low-energy implantation followed by rapid thermal annealing (RTA) results in the formation of 2-3 nm sized amorphous precipitates in a crystalline matrix. On the other hand, high-energy implantation results in an amorphous layer, with or without crystalline remnants. When the ion-beam-synthesized amorphous matrix is a continuous amorphous layer, subsequent RTA leads to the formation of 4-5 nm zinc blende (ZB)-GaN-rich crystallites in an amorphous matrix. When this matrix contains crystalline remnants, subsequent RTA leads to the formation of 2-4 nm ZB-GaN-rich crystallites within the amorphous regions. These results suggest that the matrix plays an important role in the nucleation and growth of narrow-gap nitride nanostructures, and that matrix-seeded growth may provide an opportunity to control the structure and properties of the nanostructures. (C) 2005 American Institute of Physics. [DOI: 10.1063/1.1847726]
\end{abstract}

\section{INTRODUCTION}

Recently, the controlled nanoscale crystallization of amorphous solids has emerged as a promising means for producing technologically useful nanocomposite materials. ${ }^{1}$ In the case of semiconductors, the controlled formation of crystalline structures on the nanoscale provides an opportunity for producing materials with unique electronic and optical properties. For example, due to its indirect band gap, Si has been considered unsuitable for optoelectronic applications. On the other hand, ion-beam synthesized Si nanocrystals in a $\mathrm{SiO}_{2}$ matrix exhibit significant photoluminescence and optical gains, and are therefore promising for future photonic and laser applications. ${ }^{2,3}$ Recently, we showed that a buried layer of nitrogen-rich GaAsN nanocrystallites may be synthesized within an apparently amorphous matrix by $\mathrm{N}$ ion implantation into epitaxial GaAs films followed by rapid thermal annealing (RTA). ${ }^{4}$ The GaAsN nanostructures exhibit significant photoluminescence and cathodoluminescence in the near infrared range. ${ }^{4,5}$ The apparent lowering of the fundamental band gap is likely due to the incorporation of a small amount of As in GaN. ${ }^{6}$ Similar band gap lowering is predicted for As incorporation in $\mathrm{InN}^{7}$ Therefore, ionbeam synthesis is an alternative approach for producing narrow gap nitride alloys, which are promising for a wide range of applications, including long-wavelength light-emitters and detectors, high-performance electronic devices, and high efficiency solar cells.

A few studies have been carried out on the ion-beam

\footnotetext{
a) Author to whom correspondence should be addressed; electronic mail: rsgold@umich.edu
}

synthesis of GaAsN nanostructures. ${ }^{4,8,9}$ These studies involved $\mathrm{N}$ ion implantation into GaAs substrates or $n$-doped GaAs films, with ion doses ranging from $7.5 \times 10^{15}$ to 5 $\times 10^{17} \mathrm{~cm}^{-2}$. Subsequent post-implantation thermal annealing resulted in the formation of GaN-rich nanocrystallites in either a crystalline or an amorphous matrix, depending on the ion dose. ${ }^{4,8,9}$ The structure and orientation of the GaNrich nanocrystallites are often matrix-dependent. For example, zinc blende (ZB) GaN-rich nanocrystallites formed in a crystalline GaAs matrix with essentially the same crystallographic orientations as the substrate, while randomly oriented wurtzite (WZ) crystallites formed in an amorphous GaAs matrix. ${ }^{8}$ Here, we will refer to such a matrixdependent ion-beam synthesis of nanostructures as a "matrix-seeded growth" process.

Although crystalline and amorphous matrices have been used for the matrix-seeded growth of nanostructures, the nanostructure growth in a crystalline-plus-amorphous matrix has not been investigated. In addition, matrix-seeded growth of GaAsN nanostructures using undoped epitaxial GaAs films has not been reported. Thus, the effects of dopants on the evolution of structural and optical properties of the nanostructures are not understood. Furthermore, there have been a few studies on $\mathrm{N}$ ion implantation in InAs, which focused on the effects of $\mathrm{N}$ ion implantation on the electronic properties, ${ }^{10}$ nitrogen distribution, ${ }^{11}$ damage accumulation, ${ }^{12}$ reactive ion surface etching, ${ }^{13}$ or surface nitridation. ${ }^{14}$ However, matrix-seeded growth of InAs: $\mathrm{N}$ using high energy $\mathrm{N}$ ion implantation into InAs has not been reported.

In this article, we report an investigation of the evolution of the nanometer-scale structure of nitride nanostructures synthesized using the matrix-seeded growth process, which 
High Energy N Ions

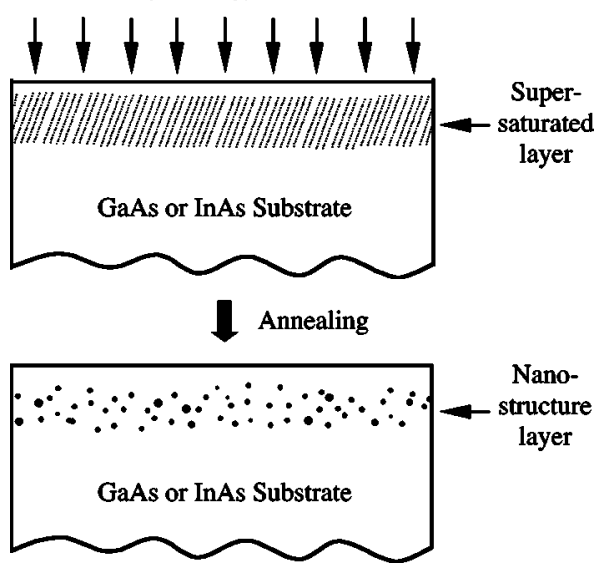

FIG. 1. Schematics of the matrix-seeded growth of nitride nanostructures: (a) high-energy $\mathrm{N}$ ions are implanted into InAs or GaAs; (b) following thermal annealing, the nitride nanostructure layer forms.

is shown schematically in Fig. 1. $\mathrm{N}$ ions are implanted into GaAs or InAs substrates or epitaxial films to form a layer supersaturated with nitrogen near the surface. Postimplantation RTA is then performed, during which N-rich nanostructures form in the nitrogen supersaturated layer. We find that low-energy implantation into GaAs and InAs substrates followed by RTA results in the formation of 2-3 nm sized amorphous precipitates in a crystalline matrix. On the other hand, high-energy implantation results in an amorphous layer, with and without crystalline remnants, in undoped and $n$-doped GaAs epitaxial films, respectively. When this matrix is a continuous amorphous layer, subsequent RTA leads to the formation of $4-5 \mathrm{~nm} \mathrm{ZB}-\mathrm{GaN}$-rich crystallites in an amorphous matrix. When the ion-beam-synthesized amorphous matrix contains crystalline remnants, subsequent RTA leads to the formation of 2-4 nm ZB-GaN-rich crystallites within the amorphous regions. These results suggest that the matrix plays an important role in the nucleation and growth of the nanostructures. In addition, dopants may enhance the amorphization of GaAs in the implantation process, as well as the growth of the crystallites during annealing.

The article is organized as follows. In Sec. II, we describe the PROFILE code simulation used for determining implantation conditions. Experimental methods for synthesizing and characterizing the nanostructures, including ion implantation, rapid thermal annealing, and transmission electron microscopy (TEM), are described in Sec. III. The structural evolution of the nitride nanostructures is presented and discussed in Sec. IV. Conclusions are given in Sec. V.

\section{PROFILE CODE SIMULATION}

To determine the implantation conditions predicted to lead to high $\mathrm{N}$ doses near the surface, we used PROFILE code simulations $^{15}$ (with sputtering coefficients determined using the Transport of Ions in Matter Code ${ }^{16}$ ). Figure 2 shows the simulation results. It is apparent that $\mathrm{N}$ ion incident energies of 50 or $100 \mathrm{keV}$, with a dose of $5 \times 10^{17} \mathrm{~cm}^{-2}$, lead to the largest near-surface retention. For example, for $\mathrm{N}$ ion implantation into GaAs (InAs), an incident ion energy of 50 $\mathrm{keV}$ and a dose of $5 \times 10^{17} \mathrm{~cm}^{-2}$ were predicted to lead to a

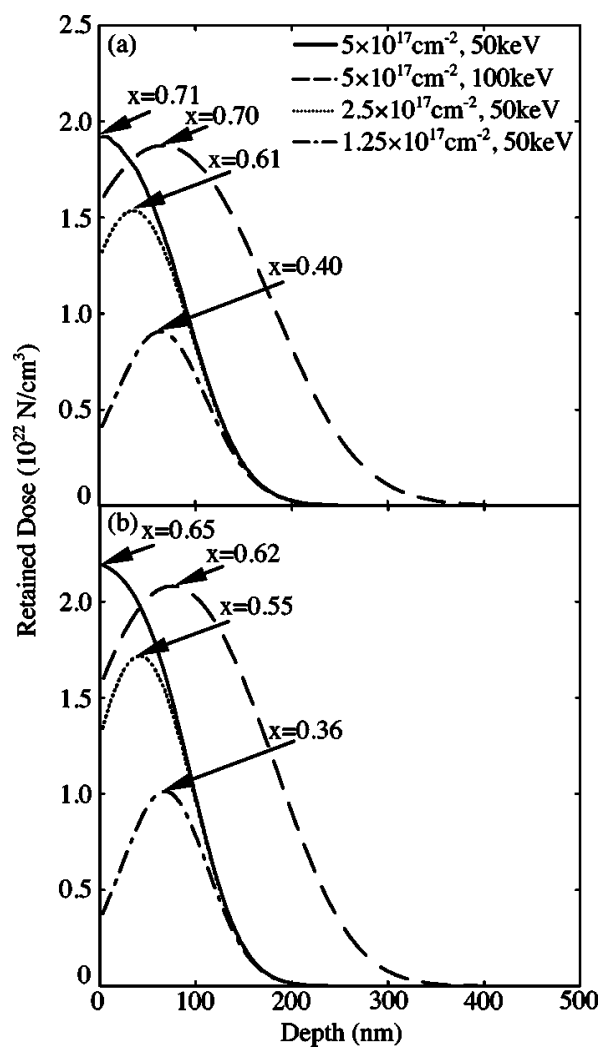

FIG. 2. PROFILE code simulations of $\mathrm{N}$ ion implanted (a) InAs and (b) GaAs, showing the retained dose of $\mathrm{N}$ as a function of depth under a variety of implantation conditions. The $x$ values indicate the maximum $\mathrm{N}$ concentration predicted by the simulations.

maximum $\mathrm{N}$ concentration of $2.2 \times 10^{22} \mathrm{~cm}^{-3} \quad(1.9$ $\times 10^{22} \mathrm{~cm}^{-3}$ ) in GaAs (InAs), located at the sample surface. The predicted total retained doses were 2.2 and 1.7 $\times 10^{17} \mathrm{~cm}^{-2}$ for GaAs and InAs, respectively. On the other hand, an incident ion energy of $100 \mathrm{keV}$ and a dose of 5 $\times 10^{17} \mathrm{~cm}^{-2}$ were predicted to lead to a maximum $\mathrm{N}$ concentration of $2.1 \times 10^{22} \mathrm{~cm}^{-3}\left(1.8 \times 10^{22} \mathrm{~cm}^{-3}\right)$, located $\sim 80 \mathrm{~nm}$ $(\sim 70 \mathrm{~nm})$ below the surface in GaAs (InAs). The predicted total retained dose for $100 \mathrm{keV}$ incident ions was 4.0 $\times 10^{17} \mathrm{~cm}^{-2}\left(3.3 \times 10^{17} \mathrm{~cm}^{-2}\right)$ for GaAs (InAs).

\section{EXPERIMENTAL PROCEDURES}

For these investigations, GaAs substrates, InAs substrates, and $\sim 2-\mu \mathrm{m}$-thick $n$-doped and undoped GaAs films grown by molecular beam epitaxy on (001) GaAs were implanted with 50 and $100 \mathrm{keV} \mathrm{N}$ ions, with a nominal dose of $5 \times 10^{17} \mathrm{~cm}^{-2}$. We will refer to the 50 and $100 \mathrm{keV}$ implantations as "low-energy" and "high-energy" implantations, respectively. To minimize channeling effects during implantation, the incident ion beam was oriented approximately $7^{\circ}$ away from the sample surface normal. The sample temperature was maintained at $300{ }^{\circ} \mathrm{C}$, as determined by an IronConstantan J-type thermocouple attached to the sample holder. The actual retained $\mathrm{N}$ doses were determined via nuclear reaction analysis (NRA) on the as-implanted samples using the reaction ${ }^{14} \mathrm{~N}\left(d, \alpha_{1}\right){ }^{12} \mathrm{C} .{ }^{17}$ For both low-energy implanted substrates, the retained $\mathrm{N}$ dose is $\sim(1.6 \pm 0.1)$ 
$\times 10^{17} \mathrm{~cm}^{-2}$. For the high-energy implanted GaAs films, the retained $\mathrm{N}$ dose is $\sim(2.9 \pm 0.1) \times 10^{17} \mathrm{~cm}^{-2}$. These NRA results are comparable to the predictions of the PROFILE code simulation presented in Sec. II.

Following implantation, some of the implanted samples were subsequently rapid thermal annealed in argon gas. Pieces of the implanted substrates were sandwiched between virgin wafers and annealed at either $800{ }^{\circ} \mathrm{C}$ for $30 \mathrm{~s}(\mathrm{GaAs})$, or at 400 or $500{ }^{\circ} \mathrm{C}$ for $10 \mathrm{~s}$ (InAs). The implanted GaAs films were annealed without a surface cap at 750,800 , or $850{ }^{\circ} \mathrm{C}$ for $30 \mathrm{~s}$. Some decomposition of the uncapped surface may occur during the annealing process. However, as shown in Fig. 2, an incident ion energy of $100 \mathrm{keV}$ and a dose $5 \times 10^{17} \mathrm{~cm}^{-2}$ were predicted to lead to a maximum $\mathrm{N}$ concentration below the surface. Therefore, the loss of $\mathrm{N}$ related to surface decomposition was expected to be minimal.

TEM imaging and electron diffraction were carried out in a JEOL 4000EX operating at $400 \mathrm{kV}$ and a JEOL 2010FX operating at $200 \mathrm{kV}$. High-resolution, bright-field, and darkfield diffraction contrast images were obtained in the JEOL 2010FX and 4000EX. Selected area diffraction (SAD) patterns were obtained in the JEOL 4000EX, using an aperture which selects a region of $\sim 0.29 \mu \mathrm{m}$ diameter, and in the JEOL 2010FX, using an aperture which selects a region of $\sim 0.18 \mu \mathrm{m}$ diameter. For each sample, an appropriate aperture was chosen in order to probe the N-rich region, as well as the GaAs or InAs matrix.

\section{RESULTS AND DISCUSSIONS}

\section{A. Low-energy implantation 1. GaAs}

Figure 3(b) shows a bright-field cross-sectional TEM image of the implanted plus $800{ }^{\circ} \mathrm{C}$ annealed GaAs substrate sample, obtained with a $\langle 220\rangle$ two-beam condition. Apparently, the low-energy implantation results in a $\sim 75$-nm-thick highly damaged surface layer, which contains nanometersized amorphous precipitates. Figure 3(c) is a high-resolution TEM (HRTEM) image collected within the surface layer, which shows some of these precipitates. The SAD pattern corresponding to Fig. 3(b) is shown in Fig. 3(a). Diffraction spots or rings related to additional phases are not apparent in the SAD pattern, suggesting a lack of crystalline GaAsN or $\mathrm{GaN}$ formed during the ion implantation and subsequent RTA.

The lack of nanocrystallites in our low-energy $\mathrm{N}$ ion implanted GaAs may be due to incomplete recrystallization as a result of the short annealing time. Indeed, nanometersized amorphous precipitates in a crystalline GaAs matrix may be transformed into GaN-rich nanocrystallites by annealing for a longer time. ${ }^{8,9}$ For example, amorphous precipitates, produced by $50 \mathrm{keV} \mathrm{N}$ ion implantation in GaAs at $380^{\circ} \mathrm{C}$, with a dose of $1.5 \times 10^{17} \mathrm{~cm}^{-2}$, transformed into crystalline $\mathrm{GaN}$ by subsequent furnace-annealing at $850^{\circ} \mathrm{C}$ for $10 \mathrm{~min}^{8}$ In another study, with $50 \mathrm{keV} \mathrm{N}$ ion implantation and a dose of $1.5 \times 10^{17} \mathrm{~cm}^{-2}$, followed by $30 \mathrm{~min}$ furnace-annealing at $700-900{ }^{\circ} \mathrm{C}$, both ZB and $\mathrm{WZ}$ GaN precipitates in a crystalline GaAs matrix were apparent. ${ }^{9}$ These

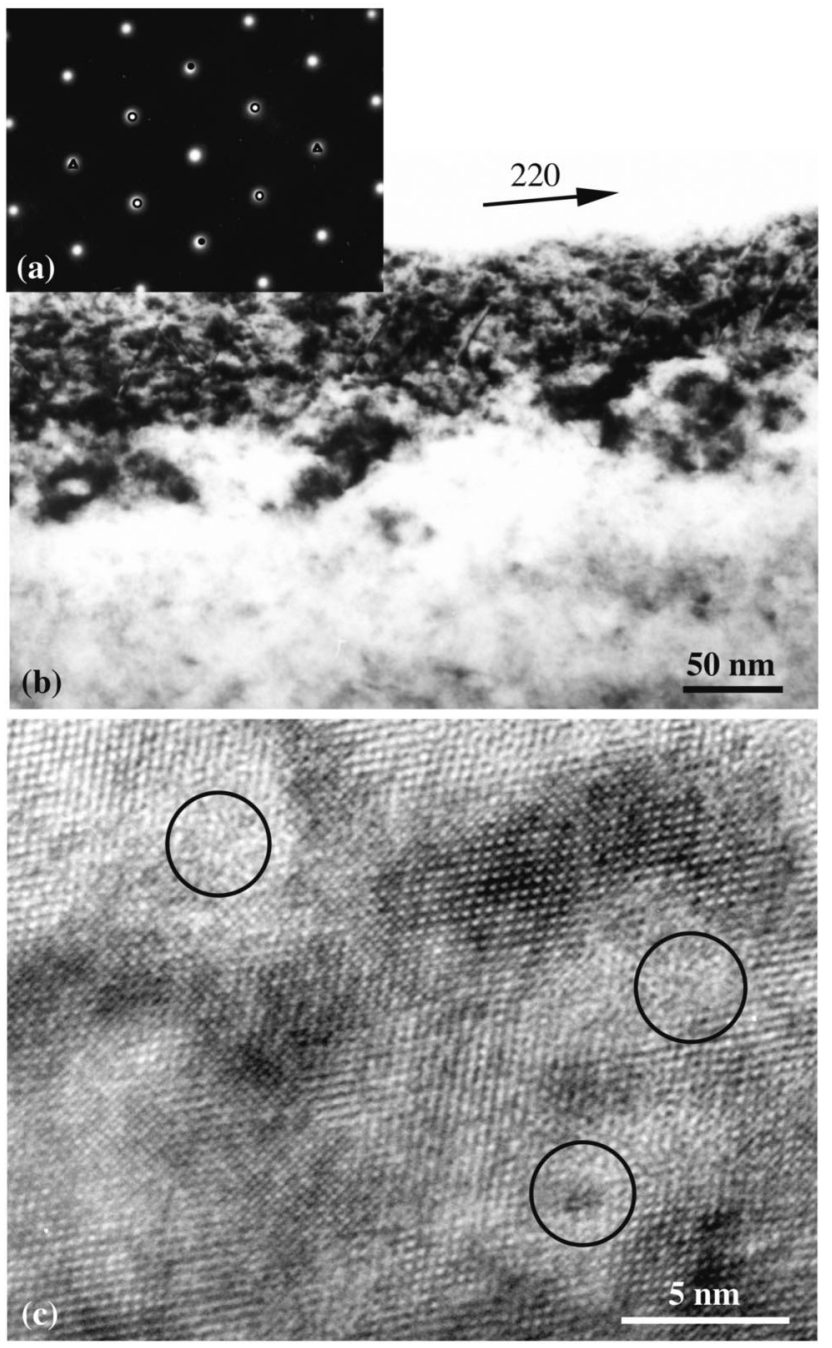

FIG. 3. (b) Bright-field cross-sectional TEM image collected using a $\langle 220\rangle$ two-beam condition and (c) HRTEM image of the implanted-plus-annealed GaAs substrate. (a) The SAD pattern corresponding to (b). The major diffraction spots of GaAs $\{111\},\{220\}$, and $\{002\}$ are indicated by circles, triangles, and black dots, respectively. Several nanometer-sized amorphous regions are circled in (c), which were collected using a $\langle 110\rangle$ zone axis.

results suggest that by controlling implantation and annealing conditions, both amorphous and crystalline GaN-rich GaAsN nanostructures in a crystalline GaAs matrix may be produced.

\section{InAs}

We also investigated the structure of low-energy $\mathrm{N}$ implanted InAs substrates using bright-field diffraction contrast TEM and HRTEM. Figure 4 shows bright-field diffraction contrast TEM images of (a) 400 and (c) $500{ }^{\circ} \mathrm{C}$ annealed samples. Both images were obtained with a $\langle 220\rangle$ two-beam condition and show evidence of three distinct regions: (1) a surface layer, (2) a middle layer, and (3) a near-substrate layer. The approximate positions of the interfaces between adjacent layers are indicated by the dashed lines. The SAD patterns corresponding to Figs. 4(a) and 4(c), which were collected from areas including all three layers, are shown in Figs. 4(b) and 4(d), respectively. Diffraction spots associated with the crystalline InAs substrate are apparent in both SAD 


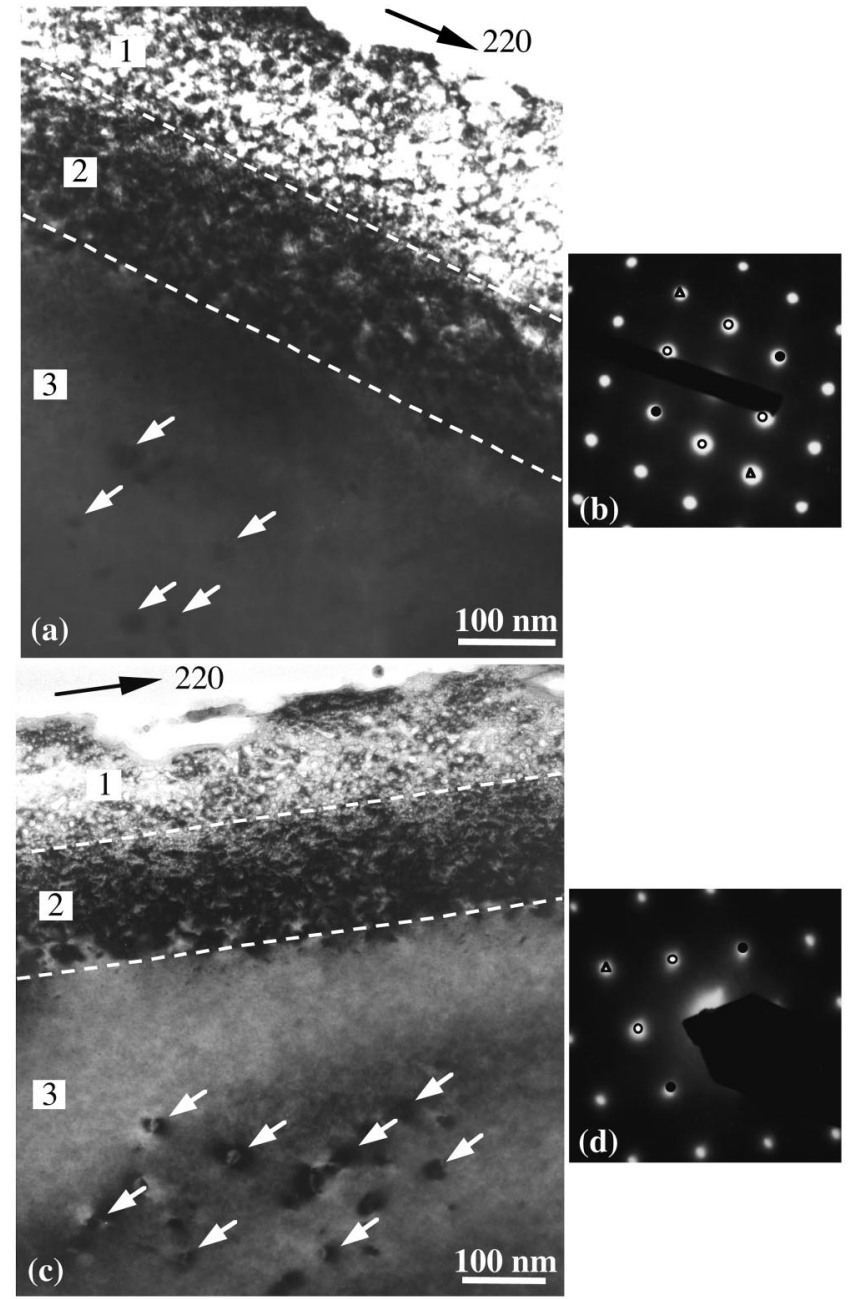

FIG. 4. Bright-field cross-sectional TEM images of implanted plus (a) 400 and (c) $500{ }^{\circ} \mathrm{C}$ annealed InAs substrates, obtained under $\langle 220\rangle$ two-beam conditions. (b) and (d) The SAD patterns corresponding to (a) and (c), respectively. The major diffraction spots of InAs $\{111\},\{220\}$, and $\{002\}$ are indicated by circles, triangles, and black dots, respectively. The white arrows in (a) and (c) indicate some typical dislocation loops.

patterns in Figs. 4(b) and 4(d). However, additional spots or rings are not evident, suggesting a lack of crystalline InAsN or InN forming during RTA.

The lack of additional crystalline phases in the implanted-plus-annealed InAs substrates is also confirmed by HRTEM. We find that the surface layer of each implanted-plus-annealed InAs sample contains a high density of amorphous precipitates, with sizes ranging from several to tens of nanometers. Figure 5(a) shows a HRTEM image of several typical amorphous precipitates in the surface layer of the $400{ }^{\circ} \mathrm{C}$ annealed InAs sample. Here, the short annealing time may have prevented the complete recrystallization of the amorphous N-rich phases. Another possibility is that the annealing temperature was too low to initiate the recrystallization process. By adjusting the annealing temperature and time to allow complete recrystallization, InAsN nanocrystallites may be obtained, similar to the case of $\mathrm{N}$ ion implanted GaAs substrates. ${ }^{8,9}$

In the middle layer of the implanted InAs substrates, complete amorphization has apparently not occurred. Instead, a high density of dislocations and $\{111\}$ planar defects
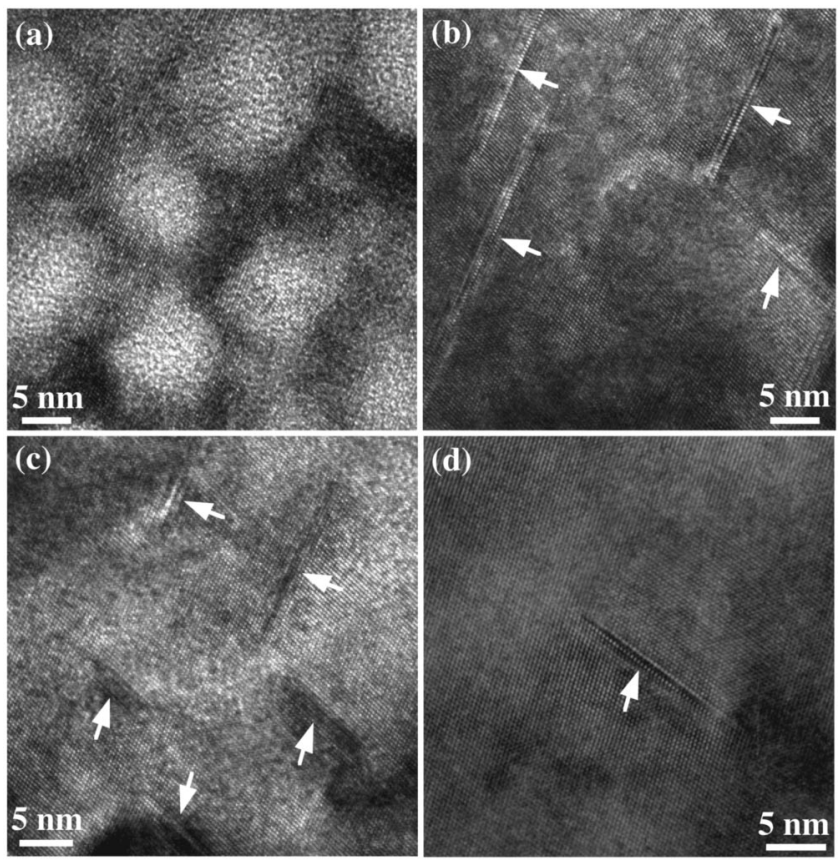

FIG. 5. HRTEM images showing (a) nanometer-sized amorphous precipitates in the InAs surface layer, (b) large $\{111\}$ planar defects in the upper middle layer, and (c) small $\{111\}$ planar defects in the lower middle layer of the $400^{\circ} \mathrm{C}$ annealed InAs, and (d) a $\{111\}$ planar defect in the near-substrate layer of the $500{ }^{\circ} \mathrm{C}$ annealed InAs. In all cases, the zone axis is $\langle 110\rangle$. The arrows in (b), (c), and (d) indicate the $\{111\}$ planar defects.

is evident. For example, Figs. 5(b) and 5(c) show HRTEM images of the $400{ }^{\circ} \mathrm{C}$ annealed InAs sample. A few typical $\{111\}$ planar defects are indicated by the arrows. The $\{111\}$ planar defects often form via the accumulation of point defects on $\{111\}$ planes during post-implantation annealing. ${ }^{18}$ Consequently, the lengths of the planar defects are expected to be dependent on damage accumulation. In an ionimplanted structure, the damage distribution is approximately Gaussian. ${ }^{19,20}$ In our $\mathrm{N}$ ion implanted InAs substrates, the position of maximum damage is likely near the surface, where the amorphous nanostructures were observed. Thus, the damage decreases with the depth, leading to a depthdependent planar defect length. Indeed, the typical planar defect length decreases from $\sim 20 \mathrm{~nm}$ near the interface between the surface and middle layers to $\sim 10 \mathrm{~nm}$ near the interface between the middle and near-substrate layers, as shown in Figs. 5(b) and 5(c), respectively.

The near-substrate layers of the implanted-plus-annealed InAs samples contain two structurally distinct regions, including a region within $\sim 200 \mathrm{~nm}$ of the middle/nearsubstrate layer interface apparently without visible extended defects, followed by a band of $\sim 10-20 \mathrm{~nm}$ particle-like features. The particle-like features are likely dislocation loops surrounding $\{111\}$ stacking faults, often observed in implanted-plus-annealed GaAs. ${ }^{18,21,22}$ For example, Fig. 5(d) shows a typical HRTEM image of a dislocation loop surrounding a $\{111\}$ stacking fault. Such dislocation loops often form via the accumulation of implantation-induced point defects on $\{111\}$ planes. ${ }^{18}$ The formation of the region without visible extended defects below the middle layer may result from the gettering of point defects by the dislocations in the 

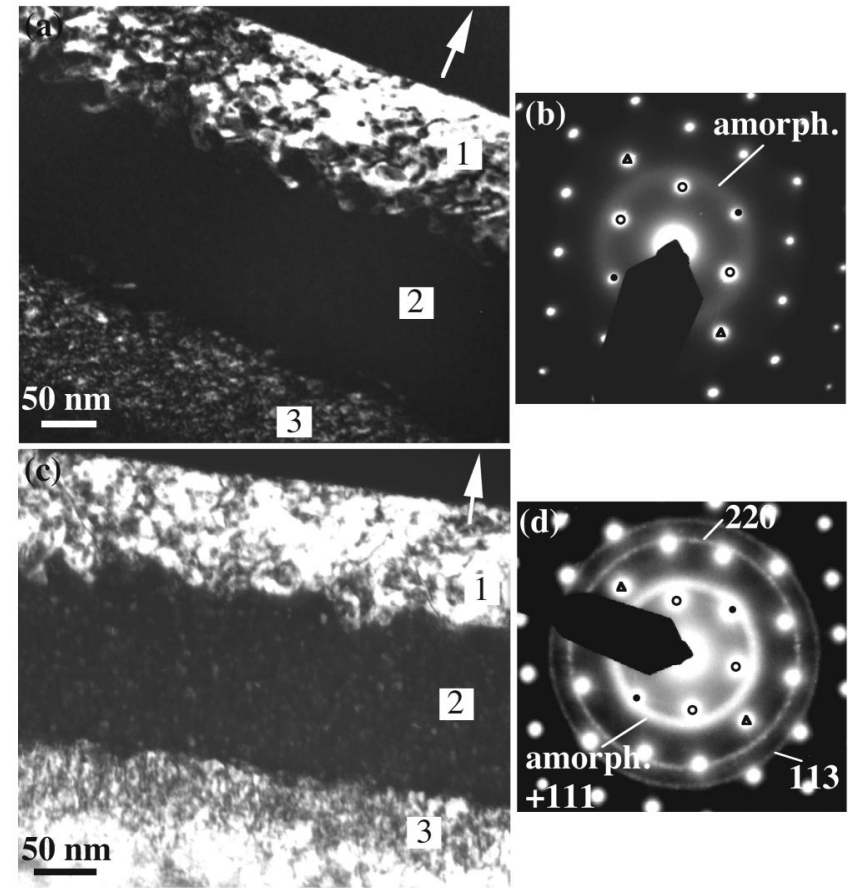

FIG. 6. Dark-field TEM images of (a) as-implanted and (c) implanted plus $800{ }^{\circ} \mathrm{C}$ annealed $n$-doped GaAs films, obtained using the GaAs 004 beam, and when possible, a portion of the crystallite 113 diffraction ring closest to the GaAs 004 spot. The arrows indicate the $\langle 004\rangle$ direction. (b) and (d) The SAD patterns corresponding to (a) and (c), respectively. The major diffraction spots from the crystalline GaAs $\{111\},\{220\}$, and $\{002\}$, are indicated by circles, triangles, and black dots, respectively.

middle layer. In implanted-plus-annealed semiconductors, regions without visible extended defects are often observed near surfaces, apparently due to the gettering of implantation-induced point defects by the surface. ${ }^{23,24}$ Since dislocations can also getter point defects in $\mathrm{GaAs},{ }^{25,26}$ the highly defective middle layer may act as a point defect sink in our case. Therefore, the implantation-induced point defects near the middle layer (within $\sim 200 \mathrm{~nm}$ ) may diffuse into the middle layer during RTA, instead of accumulating to form extended defects such as dislocation loops, ${ }^{18,21,22}$ resulting in a region apparently without visible extended defects.

\section{B. High-energy implantation}

Figures 6 and 7 show typical dark-field diffraction contrast TEM images and corresponding SAD patterns of the high-energy implanted $n$-doped and undoped GaAs films, respectively. All images were obtained using the GaAs 004 beam and when possible, a portion of the diffraction ring closest to the GaAs 004 spot. As shown in Figs. 6(a), 6(c), 7(a), and 7(c), all TEM images show evidence of three regions: (1) a surface layer, (2) 150-nm-thick middle layer, and (3) a near substrate layer. The diffraction patterns corresponding to Figs. 6(a), 6(c), 7(a), and 7(c), which were collected from areas including all three layers, are shown in Figs. 6(b), 6(d), 7(b), and 7(d), respectively.

The middle layer of the as-implanted $n$-doped GaAs film is essentially amorphous, as suggested by the opaque appearance of the middle layer shown in Fig. 6(a). This is also

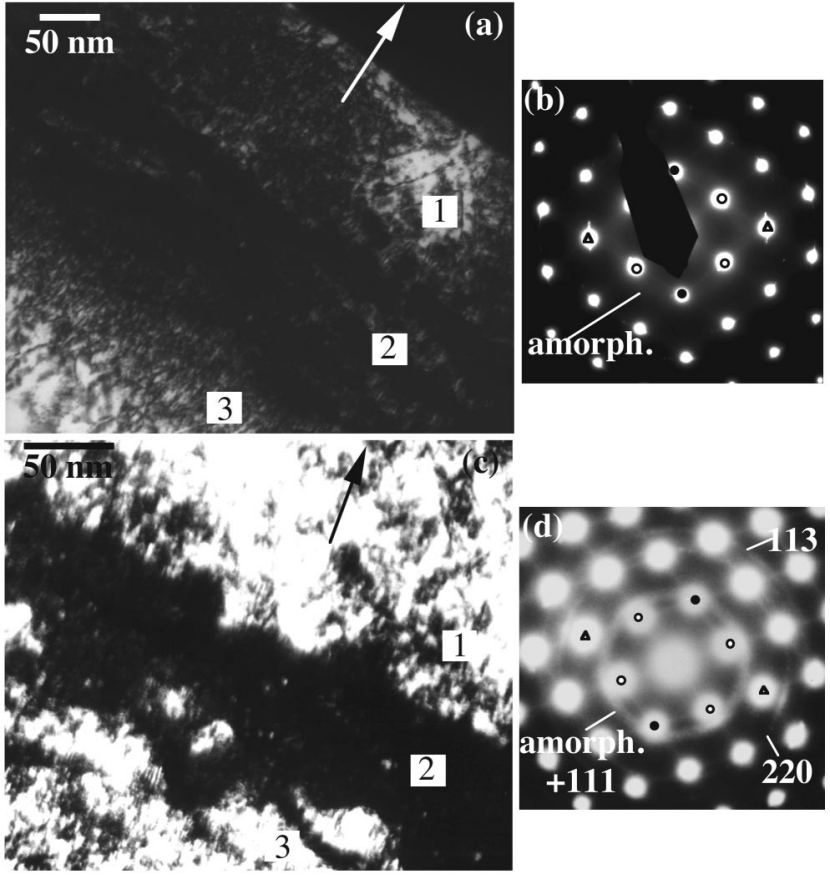

FIG. 7. Dark-field TEM images of (a) as-implanted and (c) implanted plus $800{ }^{\circ} \mathrm{C}$ annealed undoped GaAs film, obtained using the GaAs 004 beam, and when possible, a portion of the crystallite 113 diffraction ring closest to the GaAs 004 spot. The arrows indicate the $\langle 004\rangle$ direction. (b) and (d) The SAD patterns corresponding to (a) and (c), respectively. The major diffraction spots from the crystalline GaAs $\{111\},\{220\}$, and $\{002\}$, are indicated by circles, triangles, and black dots, respectively.

indicated by the SAD pattern shown in Fig. 6(b), which contains both diffraction spots associated with $\mathrm{ZB}$ GaAs, and a diffuse ring presumably due to the apparently amorphous middle layer. On the other hand, in Fig. 7(a), it is evident that the middle layer of the as-implanted undoped GaAs film contains both amorphous and crystalline phases, which appear dark and bright, respectively. Diffraction spots or rings related to additional crystalline phases are not observed in the corresponding SAD pattern shown in Fig. 7(b). This suggests that the crystalline domains in the apparently amorphous matrix of the middle layer are crystalline GaAs remnants. The coexistence of the amorphous and crystalline phases in the middle layer of the as-implanted undoped GaAs film is also suggested by the HRTEM image shown in Fig. 8(a). Similar incomplete amorphization has also been observed in As or Ga ion implanted GaAs. ${ }^{22}$

It is interesting to note that the amorphization is more complete in the $n$-doped than in the undoped GaAs film, for nominally identical implantation conditions. The enhanced amorphization in the $n$-doped GaAs film may be due to the dopants. Since amorphization often occurs via the accumulation of point defects, ${ }^{27}$ dopants may enhance the amorphization of the $n$-doped GaAs film. Another possibility is that the implantation temperature may have been higher for the undoped than that for the $n$-doped GaAs film. In GaAs, damage accumulation, which eventually leads to the amorphization, is extremely sensitive to the implantation temperature. ${ }^{28}$ For example, in Si-implanted GaAs, a temperature increase from $\sim 74$ to $80^{\circ} \mathrm{C}$ changes the residual damage from a thick amorphous layer to small disordered 

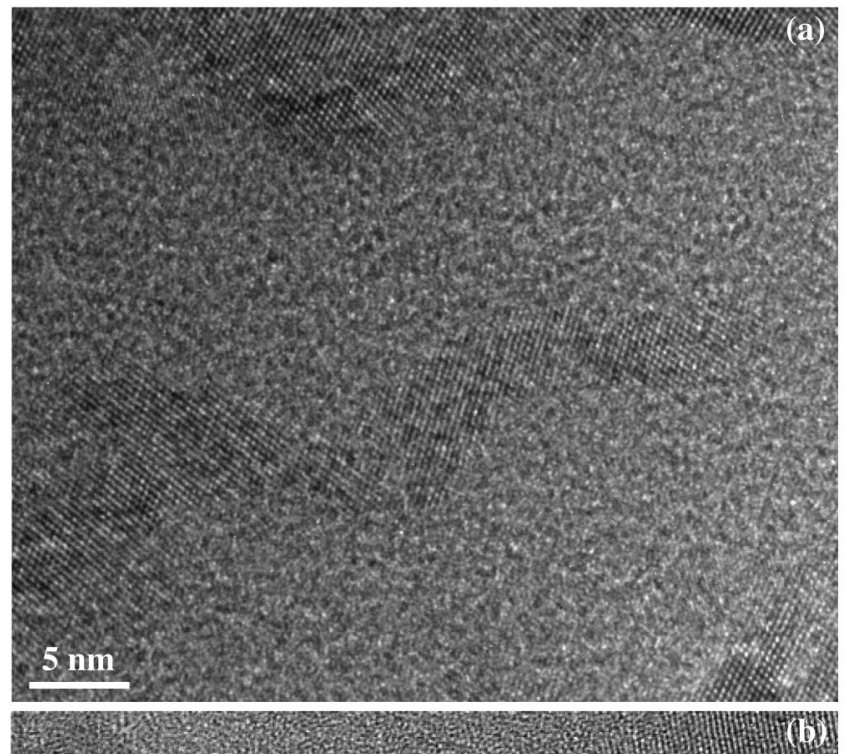

B

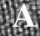

B

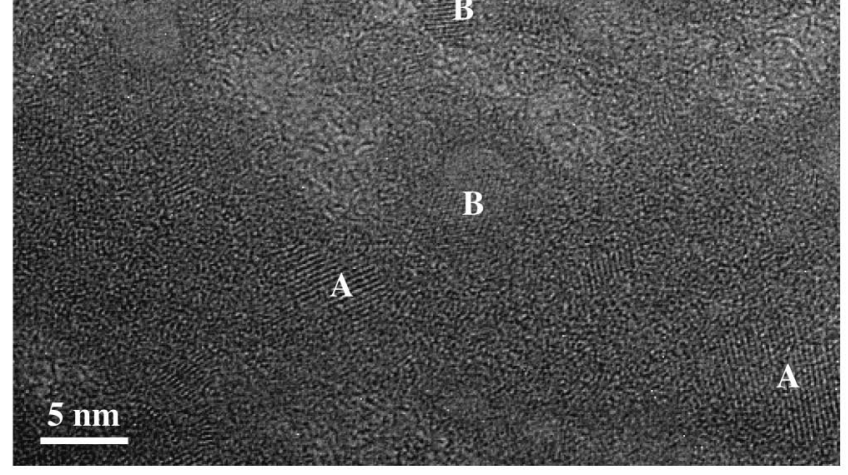

FIG. 8. High-resolution TEM images of (a) as-implanted and (b) implantedplus-annealed undoped GaAs film. In (b), GaAs remnants and GaN-rich crystallites are denoted by "A" and "B," respectively. The zone axis is $\langle 110\rangle$.

clusters. $^{28}$ This strong temperature-dependent amorphization has been explained in terms of a balance between the damage production rate and the defect annealing rate near the critical temperature for amorphization. When the implantation temperature is higher than the critical temperature for amorphization, the defect annealing rate exceeds the damage production rate, thus preventing amorphization. ${ }^{28,29}$ Thus, for the $n$-doped GaAs film, the implantation temperature is apparently lower than the critical temperature for amorphization, thus resulting in a continuous amorphous layer. On the other hand, for the undoped GaAs film, the implantation temperature might be slightly higher than the critical temperature for amorphization, leading to incomplete amorphization.

For the implanted-plus-annealed $n$-doped GaAs film, the middle layer contains a high density of nanometer-sized circular bright features, suggesting the formation of $4-5 \mathrm{~nm}$ sized nanocrystallites. ${ }^{4}$ An example is shown in the darkfield image of the $800{ }^{\circ} \mathrm{C}$ annealed sample in Fig. 6(c). The corresponding SAD pattern, shown in Fig. 6(d), reveals three diffraction rings. The interplanar spacings corresponding to these three rings are $2.61 \pm 0.01,1.60 \pm 0.01$, and $1.37 \pm 0.01 \AA$, respectively. These are within $1 \%$ of the $\{111\}$, $\{220\}$, and $\{113\}$ interplanar $d$ spacings of pure $\mathrm{ZB} \mathrm{GaN}$, suggesting the formation of GaN-rich GaAsN crystallites.

Nanometer-sized crystallites (2-4 $\mathrm{nm}$ diameter) are also apparent in the middle layer of the implanted-plus-annealed undoped GaAs films. An example is shown in Fig. 7(c), a dark-field TEM image of an implanted plus $800{ }^{\circ} \mathrm{C}$ annealed undoped film. In the corresponding SAD pattern shown in Fig. 7(d), collected from an area including all three layers, diffraction rings are apparent. The interplanar spacings corresponding to these three rings are $2.61 \pm 0.01,1.59 \pm 0.01$, and $1.36 \pm 0.01 \AA$, respectively. These are within $1 \%$ of the $\{111\},\{220\}$, and $\{113\}$ interplanar $d$ spacings of pure ZB $\mathrm{GaN}$, suggesting the formation of GaN-rich GaAsN crystallites, similar to implanted-plus-annealed $n$-doped GaAs films discussed earlier. A typical HRTEM image of the middle layer is shown in Fig. 8(b), in which two types of nanometerscale crystallites are apparent. One type of crystallite, labeled "A" in Fig. 8(b), has an average lattice fringe spacing of $\sim 3.26 \pm 0.01 \AA$, similar to $\{111\}$ interplanar spacing of GaAs. These crystallites are apparently GaAs remnants. The average lattice fringe spacing of the other type of nanocrystallite, labeled "B" in Fig. 8(b), is $\sim 2.63 \pm 0.01 \AA$, within $1.2 \%$ of the $\{111\}$ interplanar spacing of $\mathrm{ZB} \mathrm{GaN}$, further suggesting the formation of GaN-rich GaAsN crystallites.

It is interesting to note that the GaN-rich GaAsN nano crystallites in the undoped films are smaller than those in the $n$-doped films. This size difference may be due to enhanced nanocrystal growth induced by the dopants in the $n$-doped films. It is well known that dopants often increase the rate of solid phase epitaxy of $\mathrm{Si}^{30}$ Similarly, in our case, the dopants in the $n$-doped GaAs films may also play a role in enhancing recrystallization. Alternatively, the crystalline GaAs remnants in the middle layer of the undoped GaAs films may impact the GaN-rich crystallite size as follows. The GaNrich crystallites coarsen via Ostwald ripening, ${ }^{4}$ an atomic diffusion controlled process. ${ }^{31}$ Due to the increased defect density of the amorphous GaAs in comparison with its crystalline counterpart, $\mathrm{N}$ diffusion is likely faster in amorphous GaAs than in crystalline GaAs. Thus, the diffusioncontrolled coarsening of GaN-rich nanocrystallites is likely fastest in an amorphous matrix without crystalline GaAs remnants.

\section{CONCLUSIONS}

In summary, we have investigated the role of the matrix in the nucleation and growth of nitride nanostructures synthesized by nitrogen ion implantation followed by RTA. We find that low-energy implantation into GaAs and InAs substrates followed by RTA results in the formation of 2-3 nm sized amorphous precipitates in a crystalline matrix. On the other hand, high-energy implantation results in an amorphous layer, with and without crystalline remnants in undoped and $n$-doped GaAs films, respectively. Subsequent RTA leads to the formation of 2-4 nm or 4-5 nm ZB-GaNrich crystallites in an amorphous matrix with or without crys- 
talline remnants. The dopants in the $n$-doped GaAs films might have enhanced the amorphization in the implantation process, as well as the growth of the crystallites during annealing.

Our results suggest that matrix plays an important role in the nucleation and growth of nitride nanostructures. By controlling implantation conditions, e.g., implantation temperature, ion beam energy, ion doses, and dopant concentration, various matrices may be produced. Subsequently, nanostructures with various morphologies and sizes may form during post-implantation thermal annealing. Since the morphology and size of the nanostructures often greatly affect the properties of nanostructures, matrix-seeded growth provides an opportunity to produce nanostructures with unique optical and electronic properties.

\section{ACKNOWLEDGMENTS}

This work was supported in part by the U-M Office of the Vice President for Research Initiative in Materials Research Grant; the DoD Multidisciplinary University Research Initiative (MURI), administered by the Air Force Office of Scientific Research under Grant No. F49620-00-10328); the Department of Energy, through the National Renewable Energy Laboratory Photovoltaics Beyond the Horizon Program under Contract No. ACQ-1-30619-14, the TRW Foundation, and NASA-Lewis. R.C. was supported in part by DOE Grant No. DE-FG02-03ER46023. S.J.C. was supported by the NSF-REU Program through Grant No. 9773707, as well as scholarships from the Marian Sarah Parker Scholars and the General Electric Faculty for the Future Programs. We thank L.R. Berry for assistance with PROFILE code simulations, and L. Murfey and A. Bhardwaj for assistance with TEM sample preparation. The authors also acknowledge the assistance of the staff of the Michigan Ion Beam and the Electron Microbeam Analysis Laboratories at the University of Michigan. The JEOL 2010FX analytical electron microscope at EMAL is supported by NSF Grant No. 9871177.

${ }^{1}$ A. Meldrum, R. F. Haglund, Jr., L. A. Boatner, and C. W. White, Adv. Mater. (Weinheim, Ger.) 13, 1431 (2001).

${ }^{2}$ T. Shimizu-Iwayama, M. Ohshima, T. Niimi, S. Nakao, K. Saitoh, T.
Fujita, and N. Itoh, J. Phys.: Condens. Matter 5, L375 (1993).

${ }^{3}$ L. Pavesi, L. Dal Negro, C. Mazzoleni, G. Franzò, and F. Priolo, Nature (London) 408, 440 (2000)

${ }^{4}$ X. Weng, S. J. Clarke, W. Ye, S. Kumar, R. S. Goldman, A. Daniel, R. Clarke, J. Holt, J. Sipowska, A. Francis, and V. Rotberg, J. Appl. Phys. 92, 4012 (2002)

${ }^{5}$ X. Weng, R. S. Goldman, V. Rotberg, N. Bataiev, and L. J. Brillson, Appl. Phys. Lett. 85, 2774 (2004).

${ }^{6}$ L. Bellaiche, S.-H. Wei, and A. Zunger, Phys. Rev. B 54, 17568 (1996).

${ }^{7}$ N. Tit and M. W. C. Dharma-wardana, Appl. Phys. Lett. 76, 3576 (2000).

${ }^{8}$ X. W. Lin, M. Behar, R. Maltez, W. Swider, Z. Liliental-Weber, and J. Washburn, Appl. Phys. Lett. 67, 2699 (1995).

${ }^{9}$ S. Amine, G. B. Assayag, C. Bonafos, B. de Mauduit, H. Hidriss, and A. Claverie, Mater. Sci. Eng. B 93, 10 (2002).

${ }^{10}$ A. K. Sharma and P. J. Reddy, Phys. Status Solidi A 61, 295 (1980).

${ }^{11}$ A. S. Deev, P. A. Svetashov, N. A. Skakun, A. N. Grigor'ev, V. V. Ruzhitskij, V. A. Olejnik, and A. Ya. Grinchenko, Radiat. Eff. Defects Solids 114, 199 (1990).

${ }^{12}$ A. Aydinli and A. V. Drigo, Radiat. Eff. Defects Solids 113, 269 (1990).

${ }^{13}$ F. Frost, A. Schindler, and F. Bigl, Semicond. Sci. Technol. 13, 523 (1998).

${ }^{14}$ J.-D. Hecht, F. Frost, D. Hirsch, H. Neumann, A. Schindler, A. B. Preobrajenski, and T. Chassé, J. Appl. Phys. 90, 6066 (2001).

${ }^{15}$ Implantation Science Corp., Wakefield, MA 01880.

${ }^{16}$ J. Ziegler, IBM Corp.

${ }^{17}$ G. Amsel, J. P. Nadai, E. d'Artemare, D. David, E. Girard, and J. Moulin, Nucl. Instrum. Methods 92, 481 (1971).

${ }^{18}$ D. K. Sadana, T. Sands, and J. Washburn, Appl. Phys. Lett. 44, 623 (1984).

${ }^{19}$ D. K. Brice, Ion Implantation Range and Energy Deposition Distributions (Plenum, New York, 1975), Vol. 1.

${ }^{20}$ D. V. Morgan and P. D. Taylor, in Ion Implantation in Semiconductors 1976, edited by F. Chernow, J. A. Borders, and D. K. Brice (Plenum, New York, 1977), p. 557.

${ }^{21}$ J. Jasinski, K. M. Yu, W. Walukiewicz, J. Washburn, and Z. LilientalWeber, Appl. Phys. Lett. 79, 931 (2001).

${ }^{22}$ J. Jasinski, Z. Liliental-Weber, J. Washburn, H. H. Tan, C. Jagadish, A. Krotkus, S. Marcinkevicius, and M. Kaminska, J. Electron. Mater. 26, 449 (1997).

${ }^{23}$ O. W. Holland, T. P. Sjoreen, D. Fathy, and J. Narayan, Appl. Phys. Lett. 45, 1081 (1984).

${ }^{24}$ R. A. Brown and J. S. Williams, Phys. Rev. B 55, 12852 (1997).

${ }^{25}$ J. C. Bourgoin, H. J. von Bardeleben, and D. Stiévenard, J. Appl. Phys. 64, R65 (1988).

${ }^{26}$ I. Yonenaga and K. Sumino, J. Appl. Phys. 65, 85 (1989).

${ }^{27}$ F. L. Vook and H. J. Stein, Radiat. Eff. 2, 23 (1969).

${ }^{28}$ R. A. Brown and J. S. Williams, J. Appl. Phys. 81, 7681 (1997).

${ }^{29}$ R. A. Brown and J. S. Williams, J. Appl. Phys. 83, 7533 (1998).

${ }^{30}$ See, for example, O. Hellman, Mater. Sci. Eng. R 16, 1 (1996), and references therein.

${ }^{31}$ P. W. Voorhees, J. Stat. Phys. 38, 231 (1985). 